\title{
Periodontal Regeneration - Furcation Defects: Practical Applications From the AAP Regeneration Workshop
}

Mary E. Aichelmann-Reidy, ${ }^{\star}$ Gustavo Avila-Ortiz, ${ }^{\dagger}$ Perry R. Klokkevold, ${ }^{\ddagger}$ Kevin G. Murphy, ${ }^{\star \S}$ Paul S. Rosen, ${ }^{\star \|}$ Robert G. Schallhorn, ${ }^{\natural}$ Anton Sculean, ${ }^{\#}$ Hom-Lay Wang, ${ }^{\star \star}$ and Michael S. Reddy ${ }^{\dagger+}$

Focused Clinical Question: How should periodontal furcation defects be managed via periodontal regenerative therapy, and what parameters should be used for treatment selection?

Summary: The treatment of furcation defects can vary based on the type and location of the furcation involvement. Attaining predictable regenerative outcomes is dependent on the control of local and systemic factors. A combined treatment approach (barrier and bone replacement graft with or without biologic) generally offers the better therapeutic outcome over monotherapy. Class I furcation defects can be managed via conventional periodontal non-surgical and/or surgical therapy, whereas Class II furcation defects generally attain better outcomes with regenerative therapy. There is weak evidence, limited to case reports, that Class III furcation defects can be treated successfully with regenerative therapy.

Conclusions: In Class I furcation defects, regenerative therapy might be beneficial in certain clinical scenarios, although most Class I furcation defects can be treated successfully with non-regenerative therapy. For successful treatment of maxillary and mandibular molars with Class II furcation defects, systemic and local factors should be controlled, and surgical debridement and postoperative maintenance should be performed adequately. Although there is limited evidence for regeneration of Class III furcation defects, there may be a modest improvement allowing for tooth retention. Ultimately, the benefit of tooth retention and cost should be considered in the indication of therapy for teeth with severe furcation involvement. Clin Adv Periodontics 2015;5:30-39.

Key Words: Evidence-based dentistry; furcation defects; guided tissue regeneration; periodontal diseases; reconstructive surgical procedures; regeneration.

See related systematic review and consensus report in the Journal of Periodontology (February 2015, Vol. 86, No. 2s) at www.joponline.org.

\section{Background}

Treatment of teeth that present with furcation involvement represents a clinical challenge. The decision to retain and

* Department of Periodontics, School of Dentistry, University of Maryland, Baltimore, $M D$.

† Department of Periodontics, University of lowa, lowa City, IA.

‡ Department of Periodontics, School of Dentistry, University of California at Los Angeles, Los Angeles, CA.

$\S$ Private practice, Baltimore, MD.

" Private practice, Yardley, PA.

- Private practice, Aurora, CO.

\# Department of Periodontology, School of Dental Medicine, University of Bern, Bern, Switzerland.

** Department of Periodontics and Oral Medicine, School of Dentistry, University of Michigan, Ann Arbor, MI.

${ }^{+\dagger}$ Department of Periodontology, School of Dentistry, University of Alabama at Birmingham, Birmingham, AL.

Submitted September 19, 2014; accepted for publication November 17, 2014

doi: 10.1902/cap.2015.140068 treat teeth with furcations has been recognized as feasible and predictable when appropriate parameters are addressed. ${ }^{1-3}$ Moreover, the long-term prognosis of these teeth can be comparable with the survival rates of dental implants within the periodontally compromised dentition when surgically managed. Notably, survival rates of teeth affected with furcation defects after regenerative therapy ranged from $83 \%$ to $100 \%$ after an observation period of at least 5 years, according to the results of a recent systematic review. ${ }^{4}$ The treatment of furcation defects is dependent on the type and location of the defects. Essential to the successful management of furcation-involved teeth is the proper assessment of the diagnosis and prognosis relative to the identification of factors that affect or limit successful treatment. These factors may be systemic, common to all periodontal therapy, or local and specific to the anatomy of the furcation region and its proximity. Therefore, it is imperative to balance the effect of factors, be they controllable or not, with the overall patient outcome when selecting therapy. The clinician must also recognize patient-related factors. Cost, morbidity, and patient satisfaction play a role and may ultimately determine the treatment selection for furcation-involved teeth. The perceived benefit of tooth retention by some patients in severe furcation defects could be low when 


\begin{tabular}{|c|}
\hline PATIENT FACTORS \\
\hline $\begin{array}{c}\text { SYSTEMIC } \\
\text { Uncontrolled diseases and disorders } \\
\text { (e.g., diabetes, obesity, immunocompromised } \\
\text { patients) }\end{array}$ \\
\hline $\begin{array}{c}\text { BEHAVIORAL } \\
\text { Smoking, oral hygiene, patient compliance }\end{array}$ \\
\hline $\begin{array}{c}\text { PSYCHOLOGIC } \\
\text { Stress, anxiety, mental illness }\end{array}$ \\
\hline ANATOMIC AND SITE FACTORS \\
\hline Access to surgical site \\
\hline Interproximal bone height relative to furcation \\
\hline Root trunk length \\
\hline Root concavities and grooves \\
\hline Root proximity/convergence \\
\hline Furcation entrance width \\
\hline Furcation defect depth (horizontal) \\
\hline Cervical enamel projections and enamel pearls \\
\hline Tooth mobility \\
\hline Periodontal biotype \\
\hline Width of keratinized tissue \\
\hline Assessment of endodontic status \\
\hline Proximity of restorations to furcation entrance \\
\hline
\end{tabular}

FIGURE 1 A clinician checklist for evaluation of furcation-involved teeth that includes patient, local, and anatomic factors that should be assessed before the selection of appropriate regenerative therapy. expense and long-term survival are considered. This practical application describes how to manage periodontal furcation defects via periodontal regenerative therapy and the parameters used for treatment selection and modification as it relates to the level of the evidence for a predicable outcome.

The evidence supporting the treatment decisions discussed below is summarized in the recent American Academy of Periodontology Regeneration Workshop systematic review ${ }^{5}$ and consensus report. ${ }^{6}$ Several new technologies have been applied in the management of furcation defects, but limited evidence is available. These therapies are discussed in the American Academy of Periodontology Regeneration
Workshop systematic review ${ }^{7}$ and practical application ${ }^{8}$ of emerging technologies for periodontal regeneration.

\section{Decision Process}

\section{Patient Factors}

Systemic factors that limit the success of periodontal surgery must be considered when selecting any regenerative procedure. These factors may include uncontrolled diabetes and immunocompromised status or behaviors associated with compromised immune function, such as alcoholism and drug abuse, along with the associated effects of malnutrition and stress. Smoking has been identified with poor 
regenerative outcomes for furcation defects and must be considered detrimental to treatment success (Fig. 1)..$^{9}$

\section{Local and Anatomic Factors}

There are a myriad of local and anatomic factors that limit the regenerative potential of multirooted teeth (Fig. 1). Furcation caries and root fractures are considered non-modifiable, affecting not only the treatment of the furcation-involved tooth but tooth retention as well. Other factors, once modified, may only be limitations to treatment. Furcation entrance, root trunk length, and developmental abnormalities (e.g., enamel pearls, cemento-enamel projections, accessory endodontic canals, root concavities, grooves, and bifurcation ridges) may lead to disease progression within the furcation but also have the potential to influence the success of therapy, because these factors affect access necessary for adequate debridement and possibly the adaptation of barrier membranes. ${ }^{10}$ Guided tissue regeneration (GTR) outcomes are also influenced by topographic features, such as the patient's gingival tissue biotype and the presence of gingival recession. The greater the keratinized soft tissue dimension, the more likely the barrier membrane will remain covered and the greater the potential to attain clinical attachment level (CAL) gain. A thicker periodontal biotype over Class II furcation defects, defined as a bucco-lingual gingival thickness $>1 \mathrm{~mm}$, has been associated positively with minimized recession after GTR therapy compared with sites with a thinner biotype. ${ }^{11}$

Recognition of the necessity and feasibility of modification of these anatomic factors must be incorporated into the decision matrix for a multirooted tooth. ${ }^{12}$ If non-modifiable factors are present and/or access for debridement and future maintenance is not adequate, then periodontal regeneration should not be considered. For example, convergent roots are more difficult to regenerate; once furcations are affected, disease progression is favored in such situations because of the limited access for debridement. Interdental root proximity also leads to poor outcomes when proper debridement is restricted. This is particularly the case between adjacent maxillary molars and within interproximal furcation defects of maxillary molars. ${ }^{13}$ Additionally, root trunk concavities have been reported to negatively affect the outcomes of regenerative therapy, specifically for mandibular Class II furcation defects. ${ }^{14}$

Furcation characteristics. Other parameters that affect regenerative outcomes are related to the dimension and location of the furcation entrance and furcation defect configuration respective to the height of the surrounding bone. The configuration of the furcation entrance directly affects the predictability of regenerative therapy. It is worth noting that the radiographic dimensions of mandibular Class II furcation defects can be used to predict successful regeneration. Counterintuitive were the results reported by Horwitz et al. ${ }^{15}$ for mandibular Class II defects: a longer root trunk length, a furcation entrance coronal to the adjacent remaining alveolar bone crest, and a greater crestal width of the furcation appeared to negatively influence the success of outcomes when reported as horizontal CAL gains. Thus, ideally, the inter-radicular space should be sufficient to allow appropriate debridement and root preparation. However, it must be recognized that increased root divergence is associated with a larger furcation defect, which may result in reduced horizontal bone gain, furcation closure, and favorable regenerative outcome. Also, the extent of the vertical and horizontal furcal bone loss greatly affects the outcomes of GTR therapy. In general, the deeper the baseline furcation probing depth (PD), the greater the potential for horizontal furcation and vertical CAL gains, implying that deeper PDs can be used as a predictor of positive outcomes but cannot be used to predict complete furcation closure. However, it should be considered that furcation defects with $\geq 5 \mathrm{~mm}$ of vertical or horizontal bone loss usually exhibit a reduced frequency of complete clinical furcation closure. ${ }^{16}$ As the distance from the furcation roof to its base increases, the probability of furcation closure decreases. ${ }^{15,16}$ More favorable outcomes are expected in sites in which the remaining interproximal bone height is coronal to the entrance of the furcation defect when compared with those in which the bone is level or apical to the furcation entrance. ${ }^{17} \mathrm{It}$ is important to recognize that early Class II defects have the greatest frequency of clinical furcation closure after GTR therapy when observed over a 2-year period. ${ }^{16}$ Another predictable albeit non-regenerative approach for the treatment of early Class II defects is resective osseous surgery with the goal of reducing PD and the creation of more favorable anatomic contours to facilitate oral hygiene and long-term maintenance.

Furcation grade and location. Furcation classification and location are important determinants for treatment selection and regenerative success. In this discussion, the definitions of furcation degree/class are as described by Hamp et al. ${ }^{18}$ as follows: 1) Degree/Class I defect is defined as horizontal loss of periodontal tissue support $<3 \mathrm{~mm} ; 2$ ) Degree/Class II is horizontal loss of periodontal tissue support $>3 \mathrm{~mm}$ but not a through-and-through defect; and 3) Degree/Class III is a probing through-and-through defect. Proximal furcation defects may have reduced success based on the limitation for access and adaptation of barrier membranes when compared with facial and lingual molar defects. ${ }^{15}$ There is no evidence for regeneration of maxillary proximal premolar furcation defects. ${ }^{5}$ In fact, when the involved furcation entrance is $\geq 7.9 \mathrm{~mm}$ apical to the cemento-enamel junction (CEJ), ${ }^{19}$ the maxillary premolar with furcation involvement has a poor prognosis related to a compromised crown-to-root ratio. It has been established that Class I furcations, regardless of location, can be maintained successfully by non-surgical means, ${ }^{4}$ whereas deeper defects require surgery. Class II furcation defects have the strongest level of evidence for predictable outcomes after regenerative therapy, that being combination therapy with barrier and bone replacement graft or bone in combination with a biologic. ${ }^{5}$ Superior outcomes can be attained for Class II furcations receiving GTR treatment when compared with flap debridement alone. ${ }^{1-3}$ However, Class III defects have shown a variable response, with only one clinical trial reporting furcation closure of mandibular Class III 


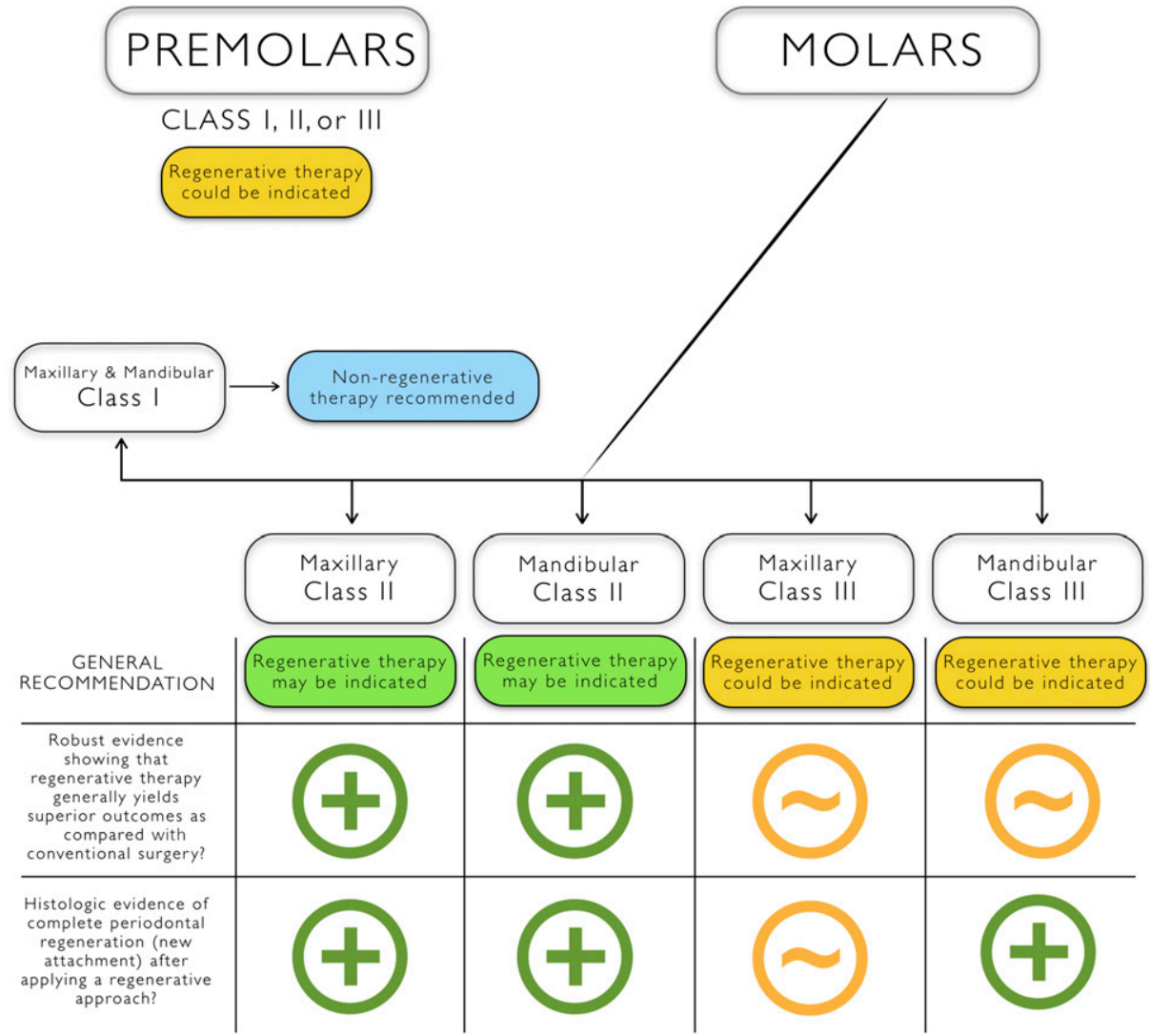

FIGURE 2 Evidence-based clinical recommendations: a treatment algorithm for an evidencebased approach to the management of furcation defects with periodontal regeneration. furcation defects. ${ }^{20}$ Most clinical trials of Class III defects reported no significant differences of treatment outcomes when comparing regenerative therapy with conventional flap surgery. ${ }^{21}$

\section{Treatment Algorithm}

Based on the evidence identified in the systematic review by Avila-Ortiz et al., ${ }^{5}$ regenerative therapy in Class II furcation defects generally leads to superior and significant vertical probing reduction and vertical and horizontal CAL gain compared with conventional flap surgery. Hence, it should be offered as the treatment of choice over extraction or conventional flap surgery, provided that there is adequate case selection and control of related etiologic factors. Mandibular furcation defects have more favorable regenerative outcomes than maxillary Class II furcation defects, and facial and lingual regenerative outcomes are greater than proximal Class II defects. ${ }^{15,22}$ Histologic evidence of periodontal regeneration has been demonstrated for maxillary and mandibular Class II furcation defects. Combination therapy, bone replacement graft, barrier or bone replacement graft and biologic, or a combination of all three, as opposed to monotherapy, produces superior results, especially when considering proximal Class II furcation defects. ${ }^{5}$ Improvement of mandibular Class III furcation defects might be possible with regenerative therapy, but evidence is limited to one clinical trial. ${ }^{20}$ Histologic evidence for partial periodontal regeneration in Class III defects is limited.
Consideration for treatment selection must be based on the type and location of the furcation defect (Fig. 2). Evaluation of the furcation bony configuration and anatomic factors must be included to determine feasibility and predictability of regenerative treatment. Recognition and modification of patient, anatomic, and local factors should be performed in conjunction with periodontal regenerative therapy for furcation defects. In preparation for regenerative therapy, measures should be made to improve plaque control and uncontrolled diabetes and to eliminate deleterious tobacco habits. Additionally, the pulpal status should be verified before the surgical visit to rule out endodontic involvement.

\section{Surgical Case Management}

The overriding principle for the management of a furcation defect (Video 1) is to attain sufficient access for thorough debridement. The flap must be wide enough to gain access for debridement and barrier membrane stabilization but as conservative as possible, as illustrated in the maxillary facial Class II case (Fig. 3). If the defect is isolated, vertical releases may be used to allow for sufficient apical movement of the flap (Video 2); otherwise, expanding the flap laterally will allow additional access. Care must be taken to preserve the keratinized tissue with sulcular incisions and full-thickness flap elevation. If insufficient soft tissue volume is present, soft tissue grafting may be required before regenerative furcation treatment or another treatment modality should be considered. Curets, files, rotary 


\section{Baseline}
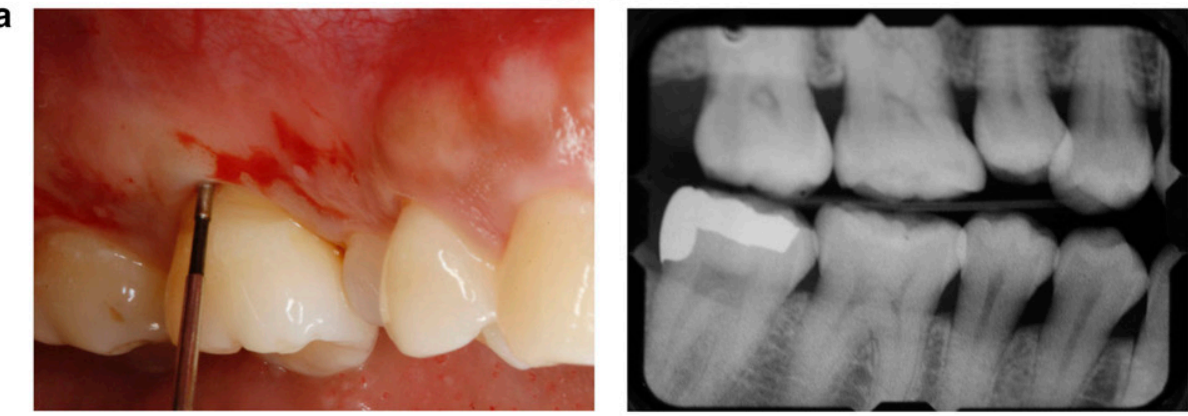

\section{Surgery}
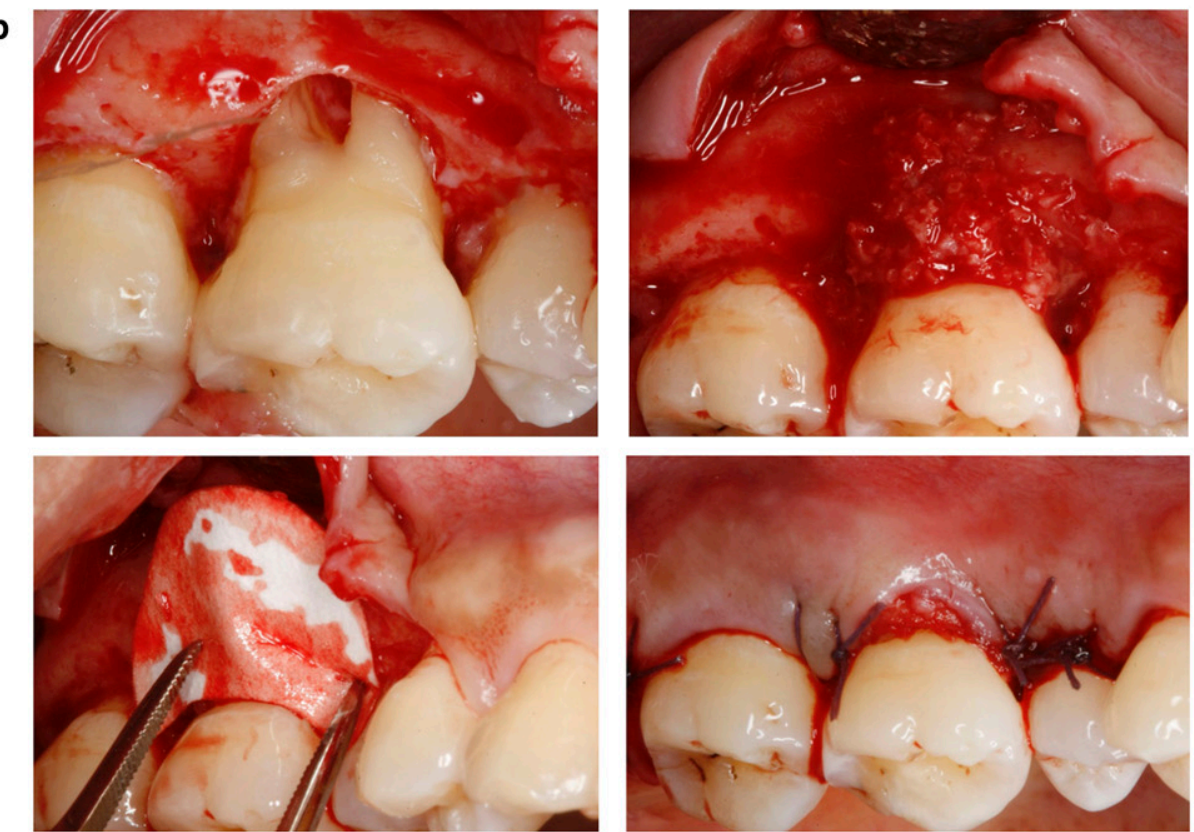

Follow-up at 2 years

c
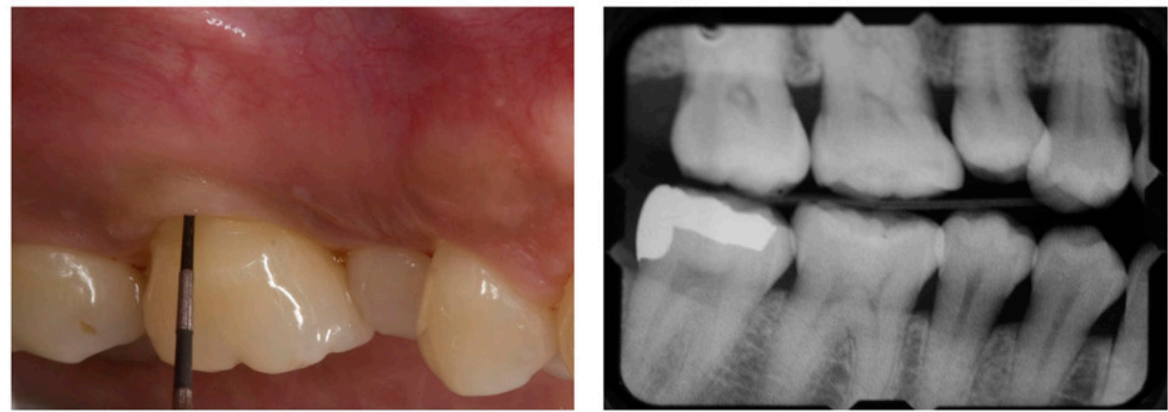

Follow-up at 4 years

d

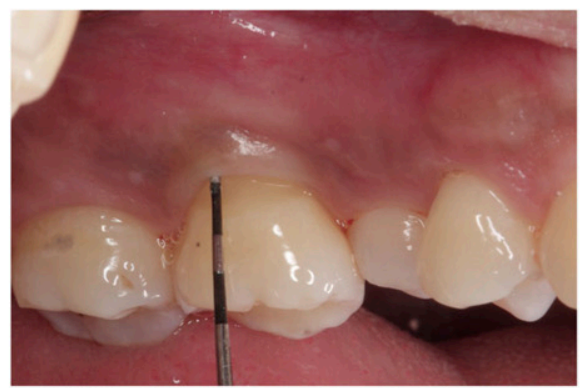

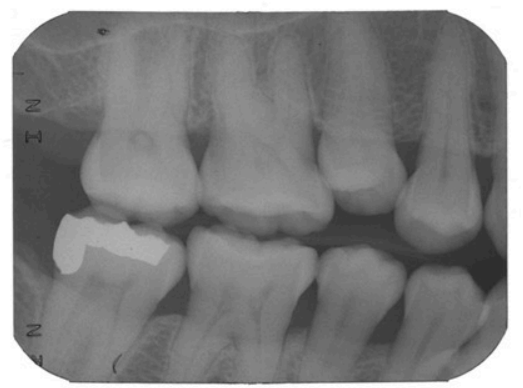

FIGURE 3 Maxillary facial Class II furcation defect. 3a This facial Class II furcation defect of the maxillary first molar displayed $6-\mathrm{mm}$ furcal PD. 3b After full-thickness flap elevation, the defect was debrided with curets, rotary instruments, and ultrasonic scalers. A freeze-dried bone allograft was adapted and condensed to the site overlaying the furcation defect and root dehiscence. A collagen barrier membrane was then contoured to extend $3 \mathrm{~mm}$ beyond the defect and adapted to the tooth and the flap and barrier were sutured together to stabilize. $3 c$ and 3d The 2- and 4-year follow-up radiographs depict bone fill, and the vertical PD was reduced to $2 \mathrm{~mm}$ from the original $6-\mathrm{mm}$ pocket. 
a

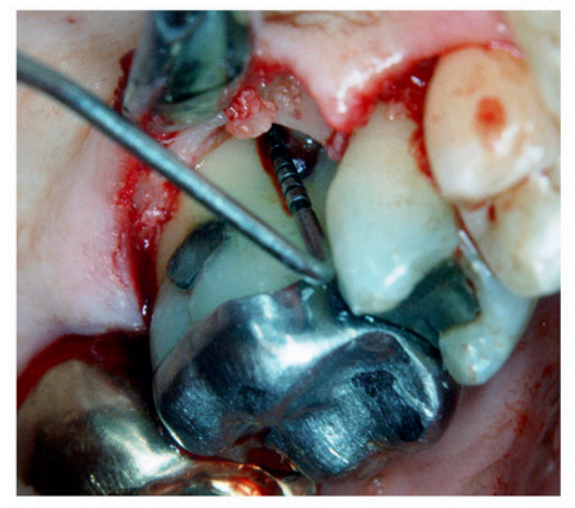

\section{Baseline}

b

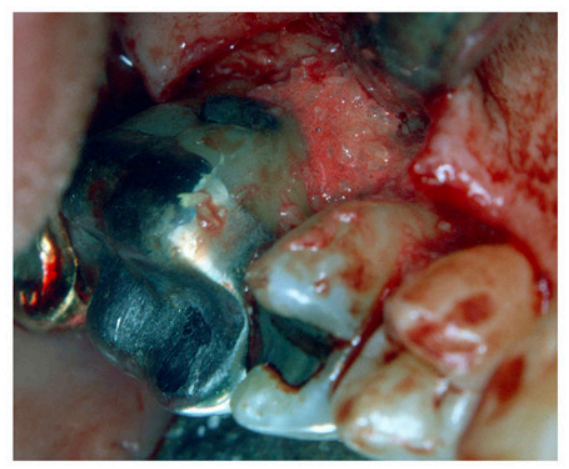

Surgery

C

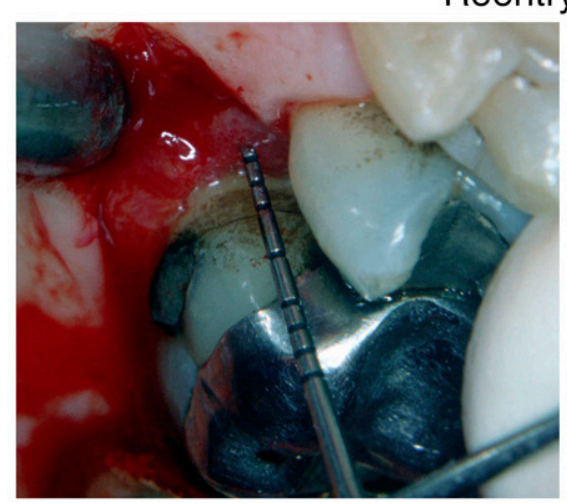

\section{Reentry at 6 weeks}

d

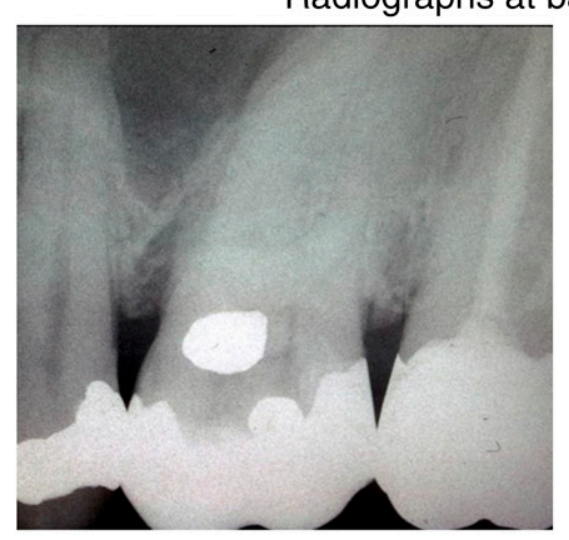

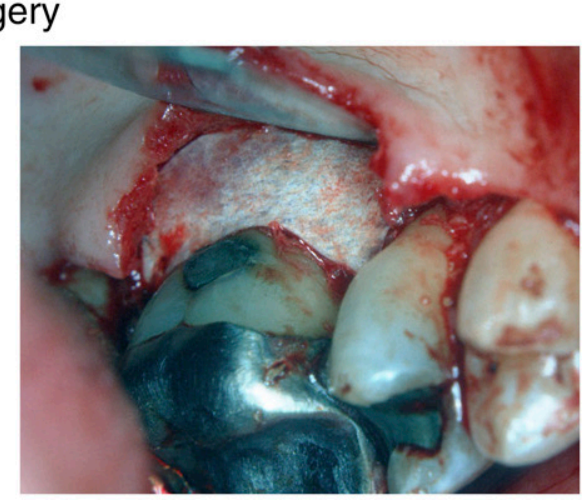
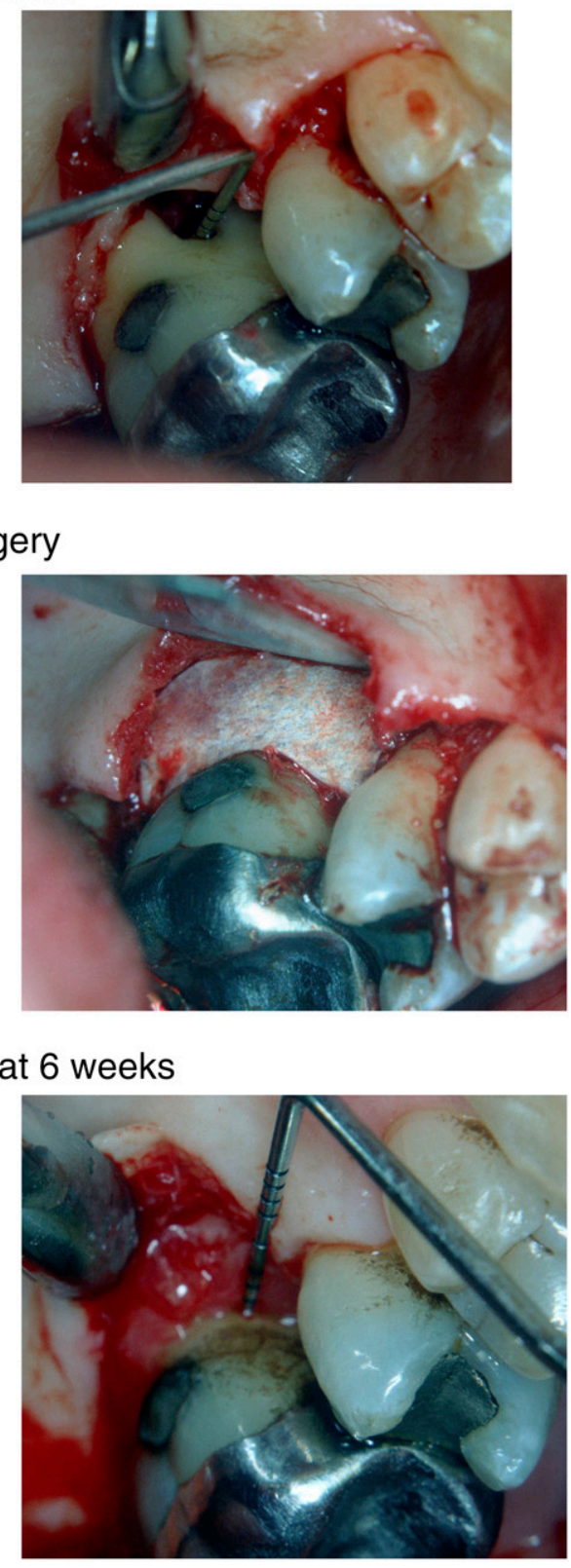

seline and 10 years

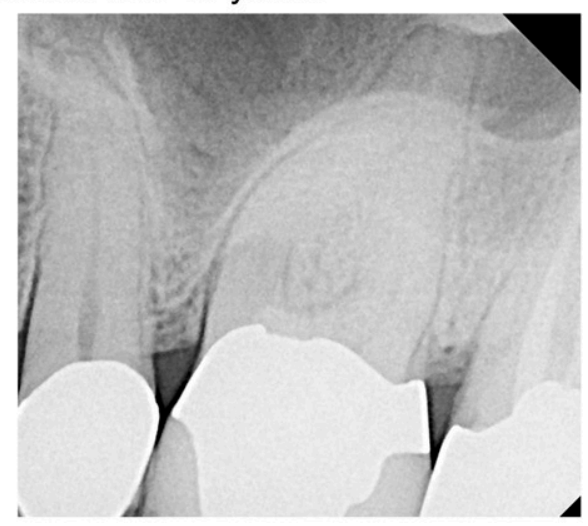

FIGURE 4 Maxillary proximal Class II furcation defect. 4a The furcation was associated with an intrabony defect with severe vertical and horizontal defect depth. 4b The furcation was debrided (smoothed) with curets, ultrasonic scalers, and rotary instruments. Note the flat, smooth topography at the furcation entrance to facilitate membrane adaptation. A demineralized freezedried bone allograft was condensed, and the expanded polytetrafluoroethylene barrier was secured to the level of the CEJ. 4c At the 6-week barrier removal, the open probing CAL was reduced. 4d The 10-year radiograph (right) reflects the treatment success and resolution of a severe Class II defect, which had $7 \mathrm{~mm}$ of horizontal attachment loss and $14 \mathrm{~mm}$ of vertical attachment loss at the initial presentation (left). 


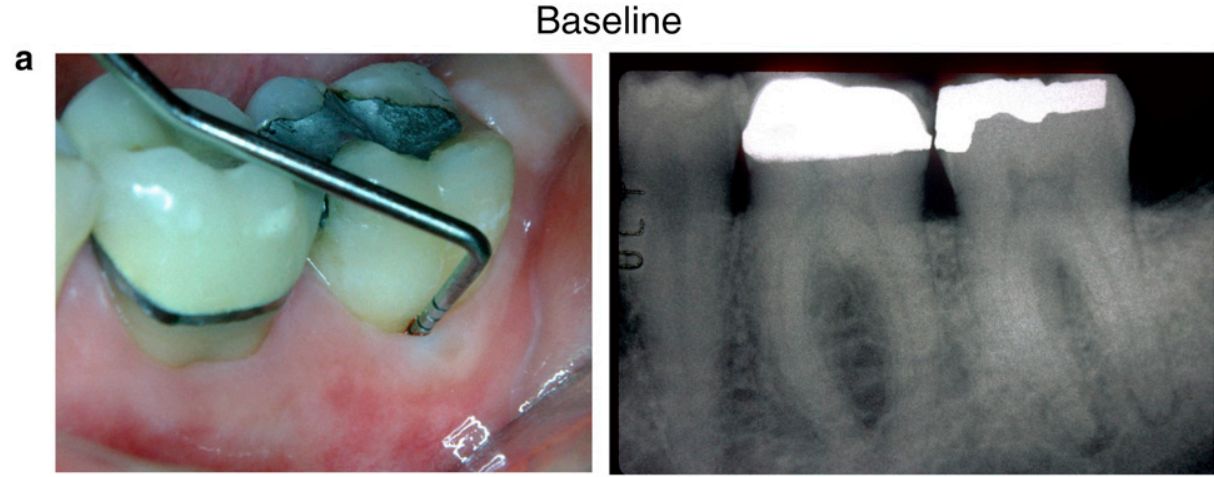

Surgery

b
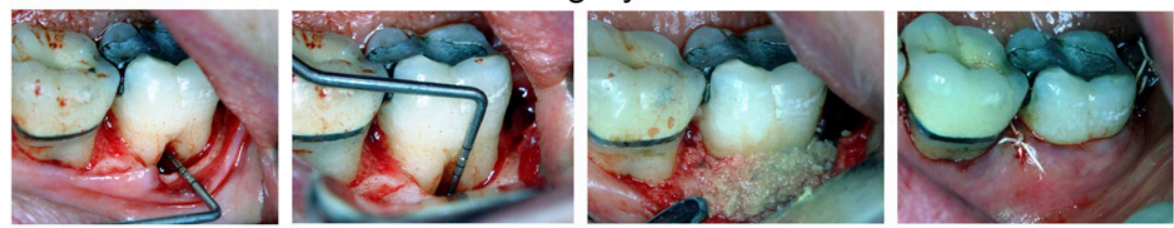

Follow-up at 12 years and 20 years

C
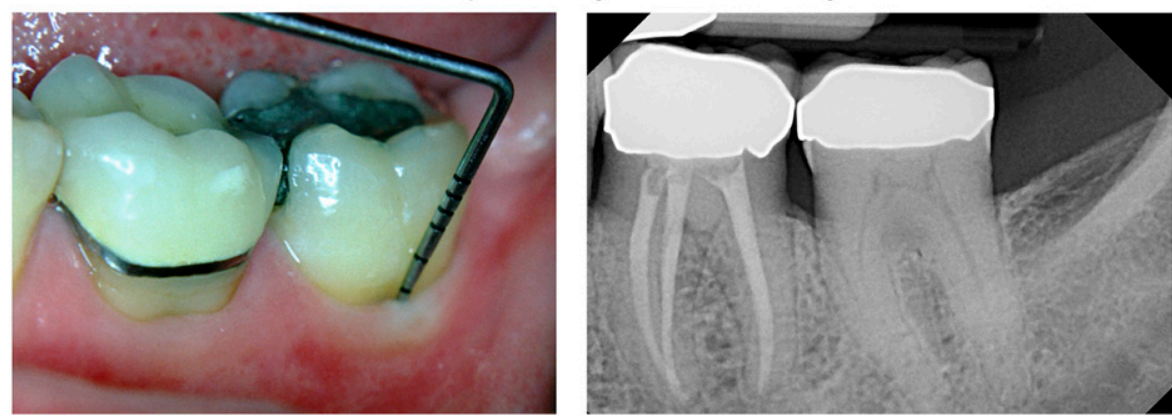

FIGURE 5 Mandibular facial Class II defect. 5a The mandibular Class II buccal furcation of the second molar had an initial 8-mm vertical PD. 5b The cervical enamel projection was removed, and the defect was subsequently debrided and then treated with citric acid before grafting with a demineralized freeze-dried bone allograft. Subsequently, an expanded polytetrafluoroethylene barrier was placed, and the flap was coronally advanced to cover the barrier. 5c Primary closure with complete coverage of the barrier was pivotal to the successful outcome demonstrated at 12 years (left). The site probed $3 \mathrm{~mm}$, and there was radiographic evidence of furcation bone fill at 20 years (right). Portions of figures $5 \mathrm{a}$ (radiograph) and $5 \mathrm{~b}$ (second image) were reproduced with permission from Quintessence (Machtei et al. ${ }^{23}$ ). instruments, and powered scalers may be necessary to debride the internal aspect of the furcation (Video 3 and Video 4). Odontoplasty should be performed by means of finishing burs to remove root anomalies, such as cemento-enamel projections, and to smooth root concavities associated with the furcation entrance. No studies to date have examined the effect of additional root conditioning on outcomes of regenerative therapy in furcation defects (Video 5). Hence, the benefit of this adjunctive root treatment during furcation regenerative treatment cannot be evaluated. The entrance of the furcation may need to be widened to gain access for the debridement. Each clinical case (Figs. 3 through 6) depicts the optimal width for instrumentation of the furcation entrance. In Fig. 4, notice the smooth, flat topography of the root trunk above the mesial entrance that was created during the root and furcation preparation. A biologic agent may be applied to the root surface or combined with graft material (Video 6). Combination therapies are recommended for successful furcation resolution. These may include but are not limited to a bone allograft or deproteinized bone matrix with a barrier of choice. Clinician selection of biomaterials, graft, and barriers does not affect the outcome, because there is a great heterogeneity of options with respect to reported horizontal bone gain and furcation closure. ${ }^{5}$ Absorbable and nonresorbable barriers alike have been used successfully. The graft must entirely fill the defect and, when possible, extend above the furcal entrance to support the barrier membrane approximating the CEJ (Figs. 3 through 6; Video 7 and Video 8).

Additionally, the barrier may be secured with sling sutures (Figs. 4 and 6; Video 9) to the tooth to promote stabilization of the wound and clot. Tension-free flap closure covering the barrier membrane is the goal (Fig. 5). The suturing technique for barrier and flap placement can be viewed in Video 10, Video 11, and Video 12. Care must be taken to limit postoperative movement of the flap. The brushing technique should be modified to not disturb the flap margin, and the patient's diet should be soft for the first 2 weeks. Observation and plaque removal should occur at 1 and 2 weeks and then monthly up to the first maintenance visit to ensure adequate plaque control. Care should be taken to not disturb the graft site during these visits. The patient will need to adhere to a 3-month maintenance schedule.

All patients in the clinical scenarios provided written or oral informed consent prior to treatment.

\section{Conclusions}

Clinical cases illustrating the necessary steps for regenerative success attained in Class II and III furcations were described. Case selection for regenerative therapy must 
a

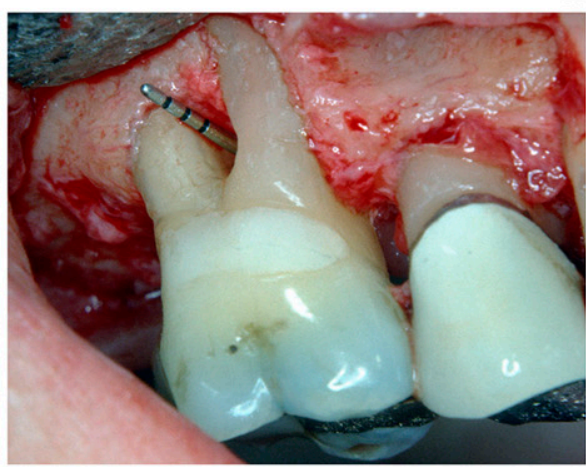

Baseline

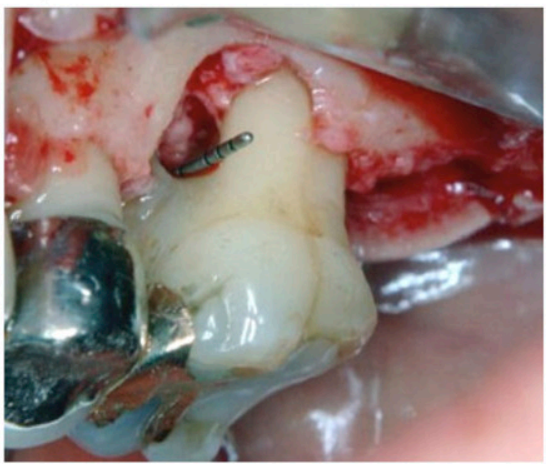

b

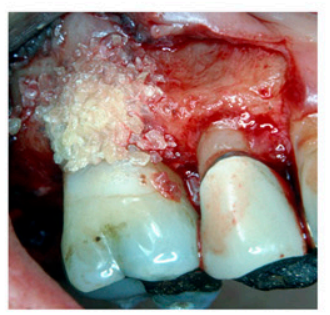

C

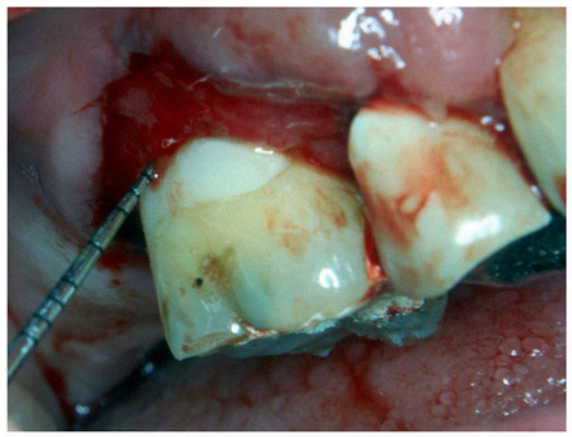

Surgery

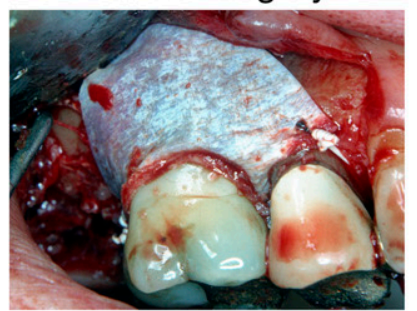

Reentry at 6 weeks
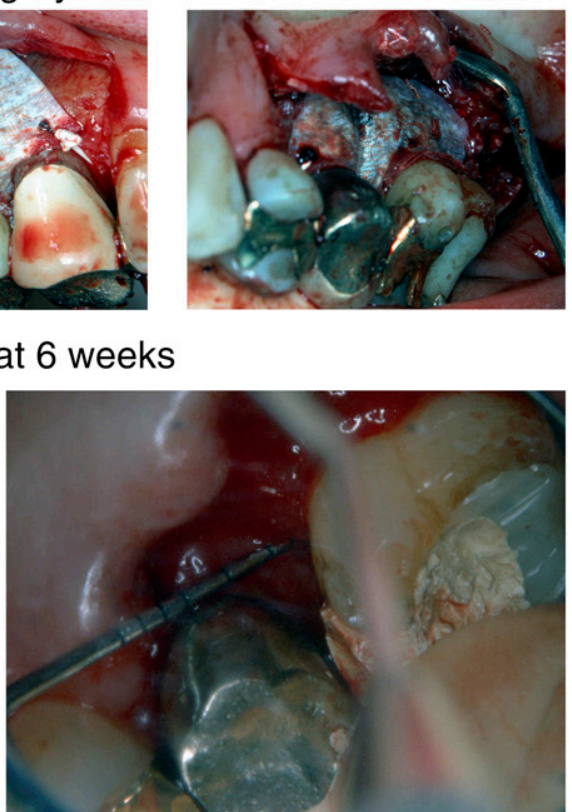

FIGURE 6 Maxillary Class III mesial to facial furcation. This case demonstrates that, if all local and anatomic factors can be managed, even a maxillary Class III furcation can be improved and the tooth retained. It is important to note that the interproximal and palatal bone levels are at the same level as the facial and mesial furcation fundus in this case, which was a key determinant in using regenerative therapy. The patient contributed to the success by following the recommended periodontal maintenance schedule. 6a The mesial and facial entrances of the defect were thoroughly debrided. The buccal root prominence was flattened. $6 \mathrm{~b}$ A demineralized freeze-dried bone allograft and expanded polytetrafluoroethylene barrier were placed to cover the furcation entrance and the root dehiscences. The barrier was secured with interproximal sling sutures, adapting it at the level of the CEJ. Note that the Class $V$ restoration on the facial aspect is appropriately contoured. 6c At the 6-week barrier membrane removal, furcation closure with immature tissue was noted. 6d The postoperative radiograph (right) illustrates the 5-year outcome with complete radiographic bone fill. Endodontic therapy was required subsequent to a pulp exposure from a restorative perforation.

\section{d Radiographs at baseline and 5 years}
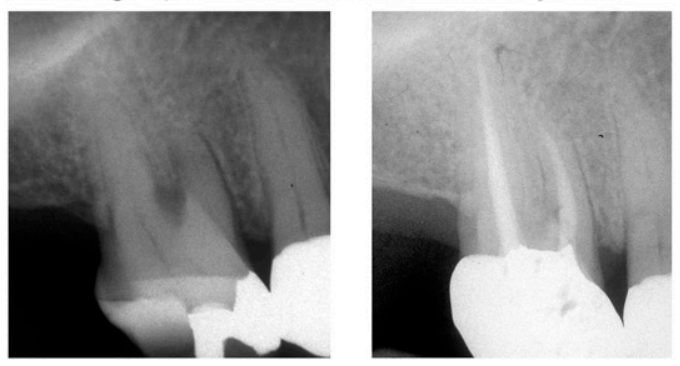

reflect the predictability and long-term prognosis of the affected tooth. Without predictability, the cost will outweigh the benefit, because multiple regenerative materials are combined to produce successful regenerative outcomes. It is recognized that Class II furcation defects are the most predictable to obtain furcation closure, whereas Class III defects will respond with gain, but furcation closure is not typically achieved. ${ }^{6}$ The gains made when associated with deeper horizontal and vertical probing could still promote partial bone fill and long-term tooth retention. In Class I furcation defects, regenerative therapy may be beneficial in certain clinical scenarios, although most Class I furcation defects may be successfully treated with non-regenerative therapy; hence, this particular scenario was not discussed in this review. None of the identified original articles reported on the application of regenerative approaches in maxillary or mandibular Class I furcation defects in molars. 


\section{Acknowledgments}

The authors thank Dr. Juan G. De Buitrago, University of Iowa, Iowa City, Iowa, for his valuable work and collaboration on the systematic review that supports some of the concepts hereby presented. Dr. Avila-Ortiz has received research funding from Osteogenics Biomedical (Lubbock, Texas), Geistlich Pharma (Wolhusen, Switzerland), DENTSPLY (York, Pennsylvania), and BioHorizons (Birmingham, Alabama) and lecture honoraria from Laboratorios INIBSA (Barcelona, Spain). Dr. Klokkevold has received research funding and/or lecture honoraria from MegaGen (Englewood Cliffs, New Jersey), J. Morita (Irvine, California), Luitpold Pharmaceuticals (Shirley, New York), Osteohealth (Shirley, New York), and Biomet 3i (Palm Beach Gardens, Florida). Dr. Murphy has received research funding from Organogenesis (Canton, Massachusetts) and Keystone Dental (Burlington, Massachusetts) and lecture honoraria from BioHorizons. Dr. Rosen has received consulting fees from Sunstar Americas (Chicago, Illinois), is on the Advisory Board of Snoasis Medical (Denver, Colorado), and is an unpaid consultant for LifeNet Health (Virginia Beach, Virginia). Dr. Sculean has received grants from Osteology Foundation (Lucerne, Switzerland), ITI Foundation (Basel, Switzerland), Botiss Dental (Berlin, Germany), Helbo (Wels, Austria), Institute Straumann (Basel, Switzerland), and Geistlich Pharma. Dr. Wang has received research funding and/or lecture honoraria from Dentium (Seoul, Korea), Neobiotech (Los Angeles, California), Zimmer Dental (Carlsbad, California), DENTSPLY, J. Morita, Osteogenics Biomedical, BioHorizons, Biomet 3i, and Botiss Dental. Dr. Reddy has received grant support and other funding through his institution, the University of Alabama at Birmingham, from BioDLogics (Memphis, Tennessee), Procter \& Gamble (Cincinnati, Ohio), Institute Straumann, Sunstar Americas, Zimmer Dental, BioHorizons, Biomet 3i, National Institutes of Health, and National Institute of Dental and Craniofacial Research. Drs. Aichelmann-Reidy and Schallhorn report no conflicts of interest related to this study. The 2014 Regeneration Workshop was hosted by the American Academy of Periodontology (AAP) and supported in part by the AAP Foundation, Geistlich Pharma North America, ColgatePalmolive, and the Osteology Foundation.
CORRESPONDENCE:

Dr. Mary E. Aichelmann-Reidy, Department of Periodontics, University of Maryland, $650 \mathrm{~W}$. Baltimore St., Baltimore, MD 21201. E-mail: mreidy@umaryland.edu. 


\section{References}

1. Jepsen S, Eberhard J, Herrera D, Needleman I. A systematic review of guided tissue regeneration for periodontal furcation defects. What is the effect of guided tissue regeneration compared with surgical debridement in the treatment of furcation defects? J Clin Periodontol 2002;29(Suppl. 3): 103-116; discussion 160-162.

2. Murphy KG, Gunsolley JC. Guided tissue regeneration for the treatment of periodontal intrabony and furcation defects. A systematic review. Ann Periodontol 2003;8:266-302.

3. Reynolds MA, Aichelmann-Reidy ME, Branch-Mays GL, Gunsolley JC. The efficacy of bone replacement grafts in the treatment of periodontal osseous defects. A systematic review. Ann Periodontol 2003;8:227-265.

4. Huynh-Ba G, Kuonen P, Hofer D, Schmid J, Lang NP, Salvi GE. The effect of periodontal therapy on the survival rate and incidence of complications of multirooted teeth with furcation involvement after an observation period of at least 5 years: A systematic review. J Clin Periodontol 2009;36:164-176.

5. Avila-Ortiz G, De Buitrago JG, Reddy MS. Periodontal regeneration Furcation defects: A systematic review from the AAP Regeneration Workshop. J Periodontol 2015;86(Suppl. 2):S108-S130.

6. Reddy MS, Aichelmann-Reidy ME, Avila-Ortiz G, et al. Periodontal regeneration - Furcation defects: A consensus report from the AAP Regeneration Workshop. J Periodontol 2015;86(Suppl. 2):S131-S133.

7. Lin Z, Rios HF, Cochran DL. Emerging regenerative approaches for periodontal reconstruction: A systematic review from the AAP Regeneration Workshop. J Periodontol 2015;86(Suppl. 2):S134-S152.

8. Rios HF, Bashutski JD, McAllister BS, et al. Emerging regenerative approaches for periodontal reconstruction: Practical applications from the AAP Regeneration Workshop. Clin Adv Periodontics 2015;5:40-46.

9. Patel RA, Wilson RF, Palmer RM. The effect of smoking on periodontal bone regeneration: A systematic review and meta-analysis. J Periodontol 2012;83:143-155.

10. Novaes AB Jr., Palioto DB, de Andrade PF, Marchesan JT. Regeneration of class II furcation defects: Determinants of increased success. Braz Dent J 2005;16:87-97.
11. Anderegg CR, Metzler DG, Nicoll BK. Gingiva thickness in guided tissue regeneration and associated recession at facial furcation defects. J Periodontol 1995;66:397-402.

12. Mardam-Bey W, Majzoub Z, Kon S. Anatomic considerations in the etiology and management of maxillary and mandibular molars with furcation involvement. Int J Periodontics Restorative Dent 1991;11:398-409.

13. Bower RC. Furcation morphology relative to periodontal treatment. Furcation entrance architecture. J Periodontol 1979;50:23-27.

14. Villaça JH, Rodrigues DC, Novaes AB Jr., Taba M Jr., Souza SL, Grisi MF. Root trunk concavities as a risk factor for regenerative procedures of class II furcation lesions in humans. J Periodontol 2004;75:1493-1499.

15. Horwitz J, Machtei EE, Reitmeir P, Holle R, Kim TS, Eickholz P. Radiographic parameters as prognostic indicators for healing of class II furcation defects. J Clin Periodontol 2004;31:105-111.

16. Bowers GM, Schallhorn RG, McClain PK, Morrison GM, Morgan R, Reynolds MA. Factors influencing the outcome of regenerative therapy in mandibular Class II furcations: Part I. J Periodontol 2003;74:1255-1268.

17. Machtei EE, Dunford RG, Norderyd OM, Zambon JJ, Genco RJ. Guided tissue regeneration and anti-infective therapy in the treatment of class II furcation defects. J Periodontol 1993;64:968-973.

18. Hamp SE, Nyman S, Lindhe J. Periodontal treatment of multirooted teeth. Results after 5 years. J Clin Periodontol 1975;2:126-135.

19. Joseph I, Varma BR, Bhat KM. Clinical significance of furcation anatomy of the maxillary first premolar: A biometric study on extracted teeth. J Periodontol 1996;67:386-389.

20. Rosen PS, Reynolds MA. Polymer-assisted regenerative therapy: Case reports of 22 consecutively treated periodontal defects with a novel combined surgical approach. J Periodontol 1999;70:554-561.

21. Pontoriero R, Lindhe J. Guided tissue regeneration in the treatment of degree III furcation defects in maxillary molars. J Clin Periodontol 1995; 22:810-812.

22. Pontoriero R, Lindhe J. Guided tissue regeneration in the treatment of degree II furcations in maxillary molars. J Clin Periodontol 1995;22: 756-763.

23. Machtei EE, Schallhorn RG. Successful regeneration of mandibular Class II furcation defects: An evidence-based treatment approach. Int J Periodontics Restorative Dent 1995;15:146-167. 